\title{
The effect of esomeprazole vs famotidine on aspirin/clopidogrel dual therapy after percutaneous coronary intervention
}

\author{
Yuanhong Wu $\mathrm{Wu}^{1, A, D-F}$, Chao Chen ${ }^{2, B, C, E, F}$, Ying Lu0 ${ }^{3, B, C, F}$, Weiwei Yu ${ }^{3, B-D}$, Shuwei Huang ${ }^{2, B-D, F}$, Dongming Lin ${ }^{2, A, E, F}$ \\ ${ }^{1}$ Department of Cardiology, Hangzhou Red Cross Hospital/Zhejiang Chinese Medicine and Western Medicine Integrated Hospital, China \\ 2 Department of Cardiology, Second Affiliated Hospital of Zhejiang Chinese Medical University, Hangzhou, China \\ ${ }^{3}$ Department of Internal Medicine, Zhejiang Chinese Medical University, Hangzhou, China \\ A - research concept and design; B - collection and/or assembly of data; C - data analysis and interpretation; \\ $\mathrm{D}$ - writing the article; $\mathrm{E}$ - critical revision of the article; $\mathrm{F}$ - final approval of the article
}

\section{Address for correspondence}

Dong-Ming Lin

E-mail:dongming_lin@163.com

Funding sources

None declared

Conflict of interest

None declared

Received on December 16, 2017

Reviewed on February 9, 2018

Accepted on February 18, 2019

Published online on October 29, 2019

Cite as

Wu Y, Chen C, Luo Y, Yu W, Huang S, Lin D. The effect of esomeprazole vs famotidine on aspirin/clopidogrel dual therapy after percutaneous coronary intervention. Adv Clin Exp Med. 2019;28(11):1519-1524. doi:10.17219/acem/104555

DOI

10.17219/acem/104555

Copyright

Copyright by Author(s)

This is an article distributed under the terms of the

Creative Commons Attribution Non-Commercial License

(http://creativecommons.org/licenses/by-nc-nd/4.0/)

\begin{abstract}
Background. Regarding drug interactions between proton pump inhibitors (PPIS) and dual antiplatelet therapy (DAPT), controversies have arisen over the possibility that PPIs may interfere with the antiplatelet effect of DAPT. However, whether this interaction is drug-specific or a class effect needs to be determined. It is not clear whether famotidine, an H2-receptor antagonist (H2RA), interacts with DAPT.

Objectives. The aim of this study was to assess the impact of esomeprazole and famotidine on the efficacy of DAPT.

Material and methods. The study involved 160 patients undergoing elective percutaneous coronary interventions and treated with DAPT and concomitant use of esomeprazole ( $40 \mathrm{mg} / \mathrm{d}$ ) or famotidine ( $40 \mathrm{mg} / \mathrm{d}$ ). Platelet reactivity was measured with adenosine diphosphate (ADP)-induced light transmittance aggregometry (LTA) and vasodilator-stimulated phosphoprotein phosphorylation-platelet reactivity index (VASP-PRI) at baseline, 14 and 30 days after applying randomized acid-suppressing agents.

Results. No significance differences were observed in treatment-by-period interactions with LTA values $(p=0.298)$ and VASP-PRI values ( $p=0.867)$, which suggested no carryover effect in either regimen over the 30-day treatment period. Intergroup comparisons were done between the 2 groups at 3 times, and similar findings were observed at each time (all $p>0.05$ ). As for intragroup measurements among the separate times, significantly lower LTA and VASP-PRI values existed on day 14 for both agents (both $p<0.05$ ).

Conclusions. The antiplatelet effect of DAPT was not affected by concomitant use of esomeprazole or famotidine. These 2 agents were much less likely than CYP2C19 polymorphisms to influence aspirin/clopidogrel therapy, supporting the assertion that the pharmacodynamic interaction between aspirin/clopidogrel and acid-suppressing agents is a drug-specific rather than a class effect.
\end{abstract}

Key words: platelet reactivity, drug-drug interaction, dual antiplatelet therapy, esomeprazole, famotidine 
Coronary heart disease (CHD) is the leading cause of death worldwide. ${ }^{1}$ Percutaneous coronary intervention (PCI) with stent implantation is increasingly used for the treatment of ischemic heart disease. In China, more than 600,000 CHD patients underwent PCI in 2016, as published in the surveillance report from the Chinese Heart Association. ${ }^{2}$ Accumulating evidence supports the utility of antiplatelet therapy as lifelong standard care following revascularization. Dual antiplatelet therapy (DAPT) consisting of aspirin and an adenosine diphosphate (ADP) receptor blocker such as clopidogrel is used to protect against thrombotic complications ${ }^{3}$; however, patients with high on-treatment platelet reactivity remain at high risk of recurrent ischemic events. ${ }^{4}$ Aspirin/clopidogrel dual antiplatelet therapy usually increases the risks of major gastrointestinal bleeding, with events increased from $0.7 \%$ in patients on aspirin alone to $1.3 \%$ in those with aspirin and clopidogrel co-therapy during 12 months of treatment. ${ }^{5,6}$ Several societies have therefore recommended acid-suppressing agents for the prevention of bleeding complications. ${ }^{7}$ Clopidogrel requires metabolism by liver cytochrome p450 enzymes (CYPs) to become an active metabolite. Among the CYPs, CYP2C19 is now regarded to play the most important role. ${ }^{8}$ Although proton pump inhibitors (PPIs) used in clinical settings are inactivated by CYP2C19, the extent of inactivation by CYP2C19 is dependent on the type of PPI; therefore, the association between PPIs and increased risk of serious cardiovascular events in patients receiving clopidogrel has led to a warning label by the FDA. ${ }^{9}$

As a frequently prescribed PPI during the maintenance of dual antiplatelet therapy, omeprazole had been reported to competitively inhibit clopidogrel transformation, resulting in decreased clopidogrel antiplatelet activity. ${ }^{9-11}$ Nevertheless, whether the clopidogrel-PPI interaction is a class effect or a drug-specific effect is still a matter of debate. $\mathrm{H}_{2}$-receptor antagonist (H2RA) has been used as an alternative to PPIs. In a previous study, H2RA was reported to be effective in the prevention of ulcers with aspirin. ${ }^{12}$ However, another study comparing PPI and H2RA regimens indicated that famotidine was inferior to pantoprazole in preventing digestive bleeding. ${ }^{13}$ Whether famotidine causes any interference with the platelet inhibitory effect of aspirin/clopidogrel is unknown. The aim of this study was to investigate the antiplatelet efficacy of DAPT with concomitant use of esomeprazole and famotidine.

\section{Material and methods}

\section{Patients and selection criteria}

We conducted this prospective, randomized trial to assess the effects of esomeprazole and famotidine on platelet inhibition by DAPT. We recruited clopidogrel-naive hospitalized patients who had been admitted to the Department of Cardiology of the Second Affiliated Hospital of Zhejiang Chinese Medical University, Hangzhou, China. The inclusion criteria included: (A) age $\geq 18$ years, (B) diagnosis of acute coronary syndromes, $(C)$ performed $\mathrm{PCI}$, and (D) received $600 \mathrm{mg}$ clopidogrel and $300 \mathrm{mg}$ aspirin loading dose between $12 \mathrm{~h}$ and $24 \mathrm{~h}$ prior to the PCI. We excluded all subjects that could be classified as having (A) class IV heart failure according to New York Heart Association (NHA) criteria, (B) thrombocytopenia (platelet count $<100 \times 10^{9} / \mathrm{L}$ ) or anemia (hemoglobin $<10 \mathrm{~g} / \mathrm{dL}$ ), (C) any chronic illness, such as cancer, liver cirrhosis or end-stage renal failure, (D) a history of hemorrhagic disorder, stroke or gastrointestinal ulcer, (E) known allergies to esomeprazole or famotidine, or who (F) refused to participate in the study.

In patients without prior antiplatelet therapy, the loading was $300 \mathrm{mg}$ of aspirin (Bayer HealthCare AG, Leverkusen, Germany) and $600 \mathrm{mg}$ of clopidogrel (Sanofi Aventis, Bridgewater, USA). Subsequently, the patients were maintained with $100 \mathrm{mg}$ of aspirin and $75 \mathrm{mg}$ daily of clopidogrel. The patients in the esomeprazole group received $40 \mathrm{mg}$ /day of esomeprazole (AstraZeneca LP, Wilmington, USA), while the famotidine group received $40 \mathrm{mg} /$ day of famotidine (Eisai Pharmaceuticals Co., Tokyo, Japan). The administration of these drugs was at the same time as the clopidogrel/aspirin. The patients were followed up daily during their hospital stay. After discharge, drug therapy compliance was assessed through a telephone call every 3 days and outpatient clinic visits on the $14^{\text {th }}$ and $30^{\text {th }}$ day. All the patients were instructed to bring their drug bags for examination.

The study complied with the Declaration of Helsinki and was approved by the Ethics Committee and Institutional Review Board of the Second Affiliated Hospital of Zhejiang Chinese Medical University. All the participants provided their written informed consent. An independent data safety monitoring committee was instituted for the adjudication of adverse clinical events.

\section{Study endpoint}

The study endpoint was residual platelet reactivity, which was assessed with the value of ADP-induced light transmittance aggregometry (LTA) and vasodilator-stimulated phosphoprotein phosphorylation-platelet reactivity index (VASP-PRI). Blood sampling for evaluating platelet function was conducted at 3 timepoints: (1) baseline (prior to randomization), (2) on the $14^{\text {th }}$ day of treatment with randomized acid-suppressing agents and (3) on the $30^{\text {th }}$ day of treatment with randomized acid-suppressing agents.

The baseline characteristics recorded included demographic data, cardiovascular risk factors and concomitant medications. All the investigators who evaluated the clinical endpoints were blinded to the results of platelet function activity. 


\section{Platelet function tests}

All platelet function tests were performed on the same day and within $2 \mathrm{~h}$ of sampling. Platelet aggregation was performed using LTA, as described in a previous study. ${ }^{14}$ In short, whole blood was centrifuged at $800 \mathrm{rpm}$ for $10 \mathrm{~min}$ to obtain platelet-rich plasma (PRP); platelet-poor plasma (PPP) was obtained by a $2^{\text {nd }}$ centrifugation of the blood fraction at 2,500 rpm for $10 \mathrm{~min}$. Light transmission was adjusted to the $100 \%$ line with PPP and a $0 \%$ baseline with PRP before the addition of the agonist. The agonist used was $20 \mu \mathrm{mol} / \mathrm{L}$ of ADP. Then, $0.45 \mathrm{~mL}$ PRP was incubated at $37^{\circ} \mathrm{C}$ for $3 \mathrm{~min}$, after which the agonist was added to the PRP. Maximal platelet aggregation (MPA) and late platelet aggregation (LPA) values (5 min after the addition of ADP) of on-treatment platelet aggregation were measured. The results were given as MPA and LPA values according to the formula:

$$
\text { [Disaggregation }(\%)=100 \times(1-\mathrm{LPA} / \mathrm{MPA})] \text {. }
$$

VASP-PRI was also performed as described in a previous study ${ }^{15}$ Briefly, citrated blood samples were incubated with prostaglandin E1 (PGE1) alone or with PGE1+ADP, and both were fixed with paraformaldehyde. After a cellular permeabilization, VASP in its phosphorylated state was labeled with a primary monoclonal antibody against serine 239-phosphorylated VASP (clone 16C2), followed by a secondary fluorescein isothiocyanate-conjugated polyclonal goat anti-mouse antibody. Final analyses were performed using quantitative flow cytometry (Biocytex Inc., Marseille, France). A platelet reactivity index (PRI) was calculated using mean fluorescence intensity (MFI) in the presence of PGE1 alone or PGE1+ADP according to the formula:

$$
\operatorname{PRI}(\%)=\left[\mathrm{MFI}_{(\mathrm{PGE} 1)}-\mathrm{MFI}_{(\mathrm{PGE} 1+\mathrm{ADP})}\right] / \mathrm{MFI}_{\mathrm{PGE} 1} \times 100 .
$$

\section{Statistical analysis}

Calculation of the sample size for the present study was based on previous experience. ${ }^{10,14}$ We estimated that a study sample size of 120 would enable detection of a one-half standard deviation (SD) difference (i.e., a 10\% difference in platelet reactivity between groups) with an $80 \%$ statistical power and a $5 \%$ alpha risk. To ensure that this sample size would be available for analysis, 60 extra patients were randomized and included. All the laboratory data was normally distributed and was described as mean \pm SD or $n$ (\%). The baseline variables of the 2 groups were compared using Pearson's $X^{2}$ test when appropriate for categorical baseline variables and the two-sample t-test for continuous baseline variables. Repeated measures analysis of variance (ANOVA) was used to evaluate the values of ADP-induced LTA and VASP-PRI at 3 points in time. ${ }^{16}$ Intergroup comparison of the respective regimens at each time point and intragroup comparison of the same regimen among the different timepoints were compared with the two-sample t-test. A p-value $<0.05$ was considered statistically significant throughout the analyses. The statistical analysis was performed using SPSS v. 22.0 software (IBM Corp., Armonk, USA).

\section{Results}

Between March and October 2017, 188 clopidogrelnaive hospitalized patients were qualified for the study according to the inclusion and exclusion criteria. Among these, 18 patients refused to participate in the study and 10 developed gastrointestinal complications prior to the initial test; therefore, a total of 160 participants were enrolled. The baseline characteristics are presented in Table 1. All the participants were randomly divided into

\begin{tabular}{|c|c|c|c|}
\hline Variables & $\begin{array}{l}\text { Esomeprazole } \\
\qquad(n=80)\end{array}$ & $\begin{array}{l}\text { Famotidine } \\
\quad(n=80)\end{array}$ & $p$-value \\
\hline Men, n (\%) & $50(62.5)$ & $48(60.0)$ & 0.746 \\
\hline Age [years] & $62.3 \pm 8.3$ & $61.8 \pm 9.1$ & 0.717 \\
\hline BMI & $29.0 \pm 5.3$ & $28.2 \pm 5.7$ & 0.359 \\
\hline \multicolumn{4}{|c|}{ Medical history, n (\%) } \\
\hline Hypertension & $32(40.0)$ & $27(33.8)$ & 0.413 \\
\hline Hyperlipidemia & $39(48.8)$ & $43(53.8)$ & 0.527 \\
\hline Current smoker & $31(38.8)$ & $28(35.0)$ & 0.623 \\
\hline Diabetes & $21(26.3)$ & $18(22.5)$ & 0.581 \\
\hline \multicolumn{4}{|c|}{$\mathrm{PCl}$ data, n (\%) } \\
\hline Chronic CAD & $23(28.8)$ & 19 (23.8) & 0.472 \\
\hline STEMI & $17(21.2)$ & $21(26.2)$ & 0.457 \\
\hline NSTEMI & $8(10.0)$ & $11(13.8)$ & 0.463 \\
\hline UA & $32(40.0)$ & $29(36.2)$ & 0.625 \\
\hline \multicolumn{4}{|c|}{ Biochemistry detection } \\
\hline $\mathrm{FBG}[\mathrm{mmol} / \mathrm{L}]$ & $6.17 \pm 1.97$ & $5.86 \pm 2.12$ & 0.340 \\
\hline $\mathrm{TG}[\mathrm{mmol} / \mathrm{L}]$ & $1.56 \pm 0.76$ & $1.71 \pm 0.83$ & 0.235 \\
\hline $\mathrm{TC}[\mathrm{mmol} / \mathrm{L}]$ & $3.70 \pm 0.91$ & $3.84 \pm 1.06$ & 0.371 \\
\hline $\mathrm{HDL}-\mathrm{C}[\mathrm{mmol} / \mathrm{L}]$ & $1.16 \pm 0.39$ & $1.05 \pm 0.32$ & 0.385 \\
\hline $\mathrm{LDL}-\mathrm{C}[\mathrm{mmol} / \mathrm{L}]$ & $2.11 \pm 0.93$ & $2.31 \pm 0.84$ & 0.155 \\
\hline $\mathrm{HbA} 1 \mathrm{C}[\%]$ & $8.27 \pm 1.88$ & $8.02 \pm 1.95$ & 0.410 \\
\hline Creatinine $[\mathrm{mmol} / \mathrm{L}]$ & $90 \pm 18$ & $93 \pm 21$ & 0.334 \\
\hline \multicolumn{4}{|c|}{ Current treatment, n (\%) } \\
\hline$\beta$-blocker & $52(65.0)$ & $46(57.5)$ & 0.330 \\
\hline Heparin & $60(100)$ & $60(100)$ & $\mathrm{N} / \mathrm{A}$ \\
\hline$C C B$ & $9(11.2)$ & $12(15.0)$ & 0.482 \\
\hline ACEI/ARB & $51(63.8)$ & $48(60.0)$ & 0.625 \\
\hline Insulin & $4(5.0)$ & $7(8.8)$ & 0.349 \\
\hline Statin & $60(100)$ & $60(100)$ & N/A \\
\hline
\end{tabular}

Table 1. Baseline characteristics of the subjects

TG - triglyceride; TC - total cholesterol; FBG - fasting blood glucose; CR - creatinine; UA - uric acid; STEMI - ST-elevation myocardial infarction; NSTEMI - non-ST-elevation myocardial infarction; UA - unstable angina; $\mathrm{BMI}$ - body mass index; $\mathrm{ACEl}$ - angiotensin-converting enzyme inhibitor; ARB - angiotensin receptor blocker; CCB - calcium channel blocker; N/A - not applicable. 


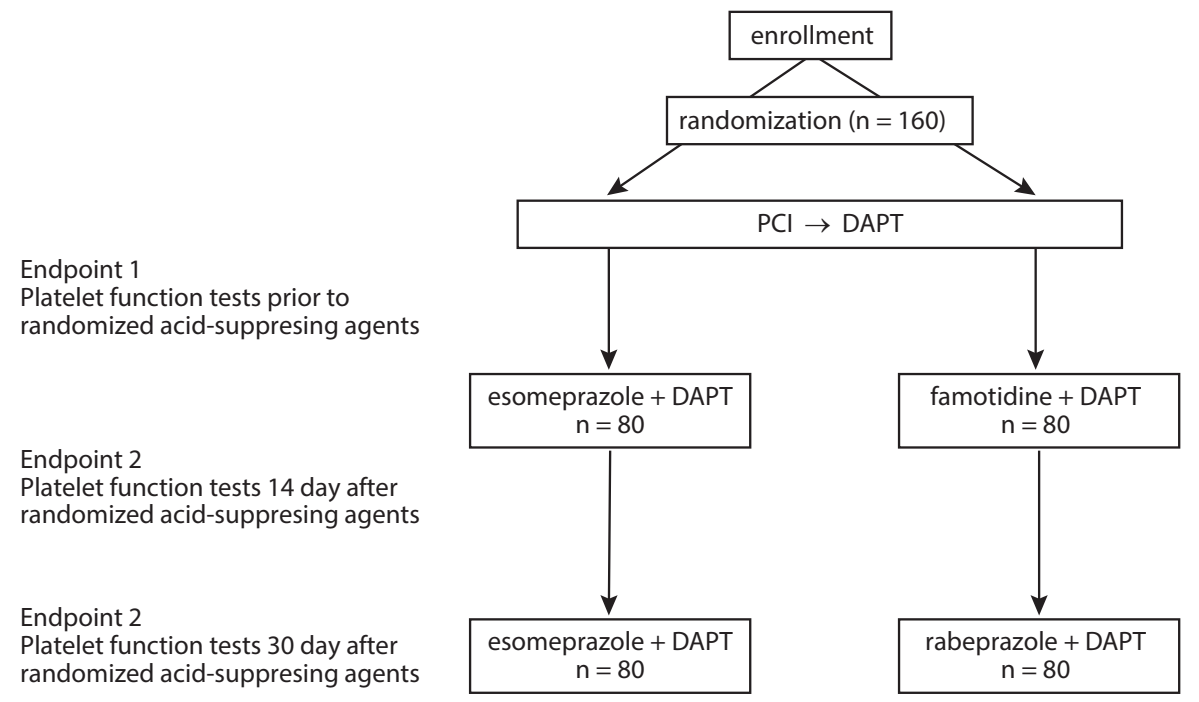

Fig. 1. Progress of the patients during the study

DAPT - dual antiplatelet therapy;

$\mathrm{PCl}$ - percutaneous coronary intervention.

Endpoint

Platelet function tests prior to randomized acid-suppresing agent

Platelet function tests 30 day after randomized acid-suppresing agents

an esomeprazole group $(\mathrm{n}=80)$ and a famotidine group $(\mathrm{n}=80)$ with the concomitant use of DAPT. The study design of the present investigation is illustrated in Fig. 1. The rate of compliance with the study drug was $100 \%$, and no patients were lost to follow-up. The baseline characteristics of both groups were comparable. None of the patients experienced bleeding or cardiac death.

Measurements were performed for ADP-induced LTA and VASP-PRI at baseline, day 14 and day 30 (Fig. 2,3). At days 14 and 30, a reduction was documented in both LTA and PRI values when compared to the baseline (both $\mathrm{p}<0.001)$. No statistically significant differences were observed in treatment-by-period interactions with LTA values $(\mathrm{p}=0.298)$ and VASP-PRI values $(\mathrm{p}=0.867)$ between the 2 regimens, which suggested no carryover effect in either regimen over the 30-day treatment period.

Intergroup comparisons between the esomeprazole and famotidine groups were done at 3 separate times (Table 2). The baseline LTA value was $41.4 \pm 7.9$ in the esomeprazole group and $40.3 \pm 6.4$ in the famotidine group $(\mathrm{p}=0.316)$, while the baseline VASP-PRI value was $68.4 \pm 11.4$

Table 2. Platelet function tests in users of esomeprazole vs famotidine at baseline and after 14 and 30 days of randomized acid-suppressing agents

\begin{tabular}{|c|c|c|c|}
\hline $\begin{array}{l}\text { Platelet function } \\
\text { test }\end{array}$ & $\begin{array}{l}\text { Esomeprazole } \\
\qquad(n=80)\end{array}$ & $\begin{array}{l}\text { Famotidine } \\
\qquad(\mathrm{n}=80)\end{array}$ & $\mathrm{p}$-value \\
\hline \multicolumn{4}{|c|}{ ADP-induced LTA [\%] } \\
\hline Baseline & $41.4 \pm 7.9$ & $40.3 \pm 6.4$ & 0.316 \\
\hline Day 14 & $35.2 \pm 6.1$ & $36.2 \pm 6.5$ & 0.330 \\
\hline Day 30 & $35.6 \pm 5.5$ & $36.8 \pm 6.4$ & 0.235 \\
\hline \multicolumn{4}{|c|}{ VASP-PRI [\%] } \\
\hline Baseline & $68.4 \pm 11.4$ & $68.3 \pm 11.9$ & 0.912 \\
\hline Day 14 & $62.2 \pm 10.5$ & $63.3 \pm 9.1$ & 0.497 \\
\hline Day 30 & $62.6 \pm 10.9$ & $63.0 \pm 9.3$ & 0.820 \\
\hline
\end{tabular}

The values are expressed as the mean $\pm S D$, unless otherwise indicated. ADP - adenosine diphosphate; LTA - light transmittance aggregometry; VASP - vasodilator-stimulated phosphoprotein; SD - standard deviation.

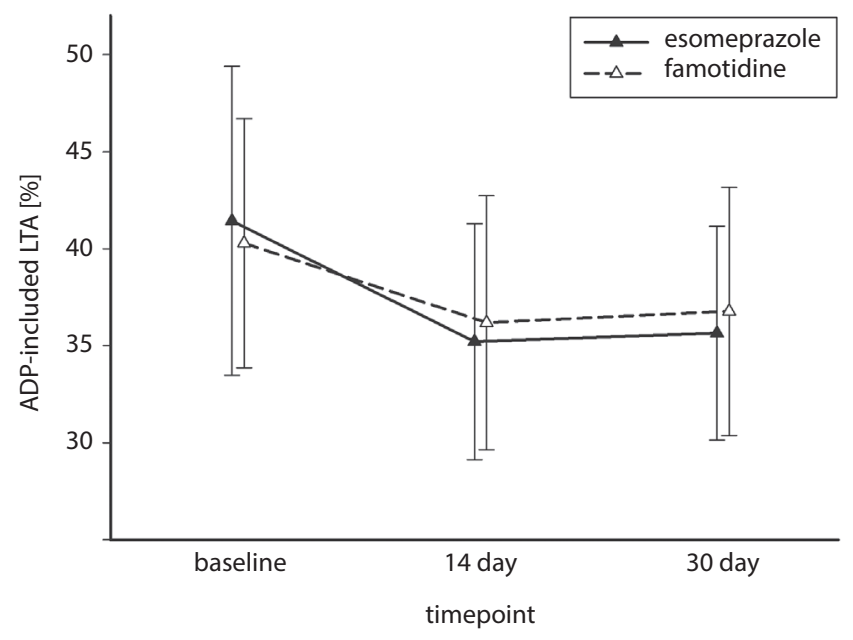

Fig. 2. $20 \mu \mathrm{mol} / \mathrm{L}$ ADP-induced LTA values across the baseline, day 14 and day 30 of randomized acid-suppressing agents

ADP - adenosine diphosphate; LTA - light transmittance aggregometry.

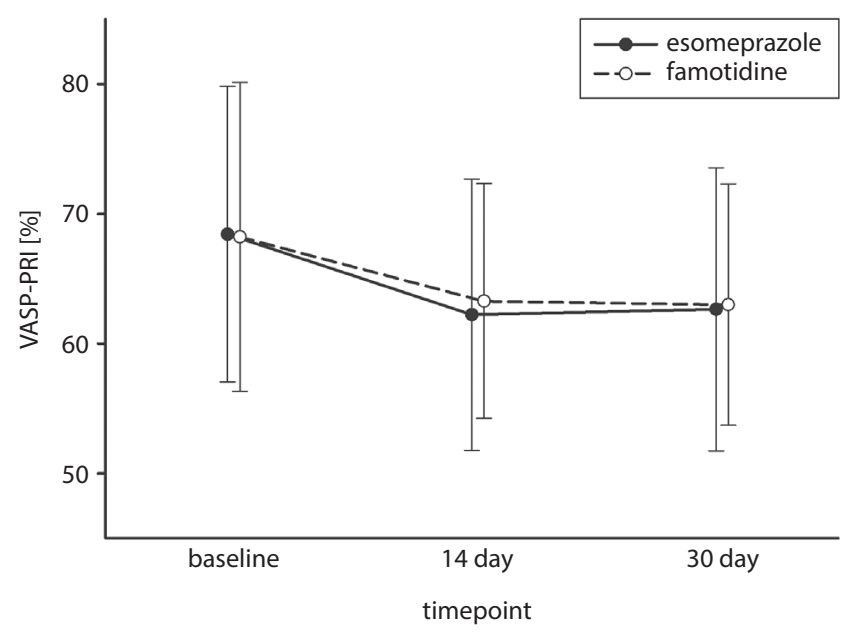

Fig. 3. VASP-PRI values across baseline, day 14 and day 30 of randomized acid-suppressing agents.

VASP - vasodilator-stimulated phosphoprotein; PRI - platelet reactivity index. 
in the esomeprazole group and $68.3 \pm 11.9$ in the famotidine group $(\mathrm{p}=0.912)$. Similar findings were also observed on day $14(\mathrm{p}=0.330$ for LTA and $\mathrm{p}=0.497$ for VASP-PRI) and day 30 ( $\mathrm{p}=0.235$ for LTA and $\mathrm{p}=0.820$ for VASP-PRI).

As for intragroup measurements among the different points in each group, statistically significant differences existed between the baseline and day 14 in both LTA (both $\mathrm{p}<0.001$ for esomeprazole and famotidine) and VASPPRI values $(\mathrm{p}<0.001$ for esomeprazole and $\mathrm{p}=0.004$ for famotidine). Moreover, comparing day 14 and day 30 of randomized acid-suppressing agents, similar LTA values $(\mathrm{p}=0.634$ for esomeprazole and $\mathrm{p}=0.567$ for famotidine) and VASP-PRI values ( $\mathrm{p}=0.805$ for esomeprazole and $\mathrm{p}=0.852$ for famotidine) were shown in the 2 groups. No cardiovascular events such as cardiovascular death, nonfatal myocardial infarction, gastrointestinal bleeding, or ischemic stroke were recorded in either group in the 30day treatment period.

\section{Discussion}

In the present prospective, randomized, case-control study, we administered esomeprazole (40 mg/day) and famotidine (40 mg/day), commonly used doses for preventing upper gastrointestinal disorders in patients receiving a combination of aspirin and clopidogrel. This study demonstrated that over the 30-day treatment period, there was no significant difference in antiplatelet effects between the 2 treatment groups. There are linear unconditionally stable results in the duration of treatment between the regimens, and no carryover effect was found in the esomeprazole or famotidine group in the setting of CHD patients receiving DAPT. Neither esomeprazole nor famotidine reduced the platelet inhibitory effect of DAPT in CHD patients who had undergone PCI. This study complements previous research, and also leads to further interest in PPIs or H2RA for upper gastrointestinal protection in DAPT users.

Concomitant use of acid-suppressing agents is usually prescribed to reduce upper gastrointestinal bleeding, but a major concern is that certain PPI drugs might abrogate antiplatelet efficiency. Because the implications correlated with the reduced pharmacodynamic effects in patients undergoing DAPT as a result of PPI drug interaction remain controversial, several studies have provided warnings against the concomitant administration of certain PPIs. ${ }^{17,18}$ Most of the available results on PPI-clopidogrel interaction is with omeprazole, a moderate CYP2C19 inhibitor, ${ }^{19,20}$ but limited data is available on the pharmacodynamic effects of other PPIs, such as esomeprazole. Because famotidine is excreted by the kidneys, hepatic enzymes (such as CYP2C19) do not metabolize such agents, and no interactions with clopidogrel and H2RAs have been reported. ${ }^{21}$ Therefore, to avoid drug-drug interaction, famotidine may be an alternative for patients treated with DAPT. Uotani et al. reported that anti-platelet drug-induced gastric injury was alleviated by famotidine without attenuation of antiplatelet functions. ${ }^{22}$ However, the sample size in their study was only 20, and the subjects were all young, healthy volunteers, not CHD patients after undergoing PCI and taking DAPT.

There have been several reports on the relationship between DAPT and the concomitant use of esomeprazole and famotidine therapy, but a definite conclusion has not yet been determined. ${ }^{23-25}$ Chan et al. showed that both treatments were comparable in preventing upper gastrointestinal bleeding in patients undergoing DAPT, ${ }^{23}$ while another study by $\mathrm{Ng}$ et al. reported that PPIs were superior to H2RAs in the prevention of upper gastrointestinal bleeding. ${ }^{24}$ Our findings were similar to the only existing study comparing the influence of the 2 agents on the platelet inhibitory effect of DAPT in which famotidine was found to have similar effects to esomeprazole during DAPT. ${ }^{25}$ However, in that report, only 88 patients completed the study protocol and they were mostly male (84\%); moreover, geographical locations and racial differences could be considered another important factor. In view of this, limited data is available on the pharmacodynamic interaction between esomeprazole and famotidine and DAPT.

This study has certain limitations. Firstly, we did not distinguish the genetic polymorphism of CYP2C19. It may be argued that CYP2C19 polymorphisms could have affected the pharmacodynamic response to clopidogrel. However, the influence of CYP2C19 loss-of-function allelic variations on clopidogrel-mediated effects is considered relatively small (5-12\%). ${ }^{8,26}$ Notably, prior studies have failed to identify any influence of CYP2C19 polymorphisms on adverse outcomes in PPI-treated patients. ${ }^{27,28}$ Secondly, this study was limited by a relatively short follow-up period, and by the cohort of average-risk DAPT users without a prior history of gastrointestinal bleeding. It has been shown that a history of upper gastrointestinal bleeding is the most important risk factor with DAPT use. Whether there are any unintended effects of concomitant use of acid-suppressing agents in longer follow-up and high-risk DAPT users should be studied further.

\section{Conclusions}

The objective of this study was to demonstrate that the concomitant use of esomeprazole or famotidine did not antagonize DAPT in Chinese patients who had undergone PCI. Our research indicates a lack of any statistically significant differences between esomeprazole and famotidine users in LTA and VASP values in treatment-by-period interaction, which suggests that neither agent had a carryover effect on DAPT during the 30-day treatment. All these results showed that esomeprazole and famotidine were much less likely than CYP2C19 to influence the patients' 
response to clopidogrel, indicating that the pharmacodynamic interaction between clopidogrel and acid-suppressing agents is a drug-specific effect rather than a class effect.

\section{References}

1. Halvorsen S, Storey RF, Rocca B, et al; ESC Working Group on Thrombosis. Management of antithrombotic therapy after bleeding in patients with coronary artery disease and/or atrial fibrillation: Expert consensus paper of the European Society of Cardiology Working Group on Thrombosis. Eur Heart J. 2017;38(19):1455-1462.

2. Zhang GS, Yu CH, Luo LS, Li YC, Zeng XY. Trend analysis of the burden of ischemic heart disease in China, 1990 to 2015 [in Chinese]. Zhonghua Yu Fang Yi Xue Za Zhi. 2017;51(10):915-921.

3. Angiolillo DJ, Rollini F, Storey RF, et al. International Expert Consensus on Switching Platelet P2Y12 Receptor-Inhibiting Therapies. Circulation. 2017;136(20):1955-1975.

4. Bonello L, Harhouri K, Sabatier F, et al. Level of adenosine diphosphate receptor P2Y12 blockade during percutaneous coronary intervention predicts the extent of endothelial injury, assessed by circulating endothelial cell measurement. J Am Coll Cardiol. 2010;56(13): 1024-1031.

5. Hearnshaw SA, Logan RF, Lowe D, Travis SP, Murphy MF, Palmer KR. Acute upper gastrointestinal bleeding in the UK: Patient characteristics, diagnoses and outcomes in the 2007 UK audit. Gut. 2011;60(10): 1327-1335.

6. Lewis JD, Bilker WB, Brensinger C, Farrar JT, Strom BL. Hospitalization and mortality rates from peptic ulcer disease and GI bleeding in the 1990s: Relationship to sales of nonsteroidal anti-inflammatory drugs and acid suppression medications. Am J Gastroenterol. 2002;97(10):2540-2549.

7. Bhatt DL, Scheiman J, Abraham NS, et al; American College of Cardiology Foundation Task Force on Clinical Expert Consensus Documents. ACCF/ACG/AHA 2008 expert consensus document on reducing the gastrointestinal risks of antiplatelet therapy and NSAID use: A report of the American College of Cardiology Foundation Task Force on Clinical Expert Consensus Documents. Circulation. 2008:118(18):1894-1909.

8. Shuldiner AR, O'Connell JR, Bliden KP, et al. Association of cytochrome P450 2C19 genotype with the antiplatelet effect and clinical efficacy of clopidogrel therapy. JAMA. 2009;302(8):849-857.

9. Bhatt DL, Cryer BL, Contant CF, et al; COGENT Investigators. Clopidogrel with or without omeprazole in coronary artery disease. $N$ Engl J Med. 2010;363(20):1909-1917.

10. Gilard M, Arnaud B, Cornily JC, et al. Influence of omeprazole on the antiplatelet action of clopidogrel associated with aspirin: The randomized, double-blind OCLA (Omeprazole Clopidogrel Aspirin) study. J Am Coll Cardiol. 2008;51(3):256-260.

11. Ho PM, Maddox TM, Wang $L$, et al. Risk of adverse outcomes associated with concomitant use of clopidogrel and proton pump inhibitors following acute coronary syndrome. JAMA. 2009;301(9):937-944.

12. Taha AS, McCloskey C, Prasad R, Bezlyak V. Famotidine for the prevention of peptic ulcers and oesophagitis in patients taking low-dose aspirin (FAMOUS): A phase III, randomised, double-blind, placebocontrolled trial. Lancet. 2009;374(9684):119-125.

13. $\mathrm{Ng} \mathrm{FH}$, Wong SY, Lam KF, et al. Famotidine is inferior to pantoprazole in preventing recurrence of aspirin-related peptic ulcers or erosions. Gastroenterology. 2010;138(1):82-88.
14. Liu LP, Wang Y, Si R, Yuan M, Cheng K, Guo WY. Esomeprazole and rabeprazole did not reduce antiplatelet effects of aspirin/clopidogrel dual therapy in patients undergoing percutaneous coronary intervention: A prospective, randomized, case-control study. Expert Opin Pharmacother. 2016;17(1):7-16.

15. Tsantes A, Ikonomidis I, Papadakis I, et al. Evaluation of the role of the new INNOVANCE PFA P2Y test cartridge in detection of clopidogrel resistance. Platelets. 2012;23(6):481-489.

16. Gliner JA, Morgan GA, Harmon RJ. Single-factor repeated-measures designs: Analysis and interpretation. J Am Acad Child Adolesc Psychiatry. 2002;41(8):1014-1016.

17. Fernando H, Dart AM, Peter K, Shaw JA. Proton pump inhibitors, genetic polymorphisms and response to clopidogrel therapy. Thromb Haemost. 2011;105(6):933-944.

18. Ferreiro JL, Ueno M, Capodanno D, et al. Pharmacodynamic effects of concomitant versus staggered clopidogrel and omeprazole intake: Results of a prospective randomized crossover study. Circ Cardiovasc Interv. 2010;3(5):436-441.

19. Frelinger AL, Bhatt DL, Lee RD, et al. Clopidogrel pharmacokinetics and pharmacodynamics vary widely despite exclusion or control of polymorphisms (CYP2C19, ABCB1, PON1), noncompliance, diet, smoking, co-medications (including proton pump inhibitors), and pre-existent variability in platelet function. J Am Coll Cardiol. 2013; 61(8):872-879.

20. Kwok CS, Jeevanantham V, Dawn B, Loke YK. No consistent evidence of differential cardiovascular risk amongst proton-pump inhibitors when used with clopidogrel: Meta-analysis. Int J Cardiol. 2013;167(3): 965-974.

21. Hibma JE, Zur AA, Castro RA, et al. The effect of famotidine, a MATE1selective inhibitor, on the pharmacokinetics and pharmacodynamics of metformin. Clin Pharmacokinet. 2016;55(6):711-721.

22. Uotani T, Sugimoto $M$, Nishino $M$, et al. Prevention of gastric mucosal injury induced by anti-platelet drugs by famotidine. J Clin Pharmacol. 2014;54(8):858-864.

23. Chan FK, Kyaw M, Tanigawa T, et al. Similar efficacy of proton-pump inhibitors vs $\mathrm{H} 2$-receptor antagonists in reducing risk of upper gastrointestinal bleeding or ulcers in high-risk users of low-dose aspirin. Gastroenterology. 2017;152(1):105-110.e1.

24. $\mathrm{Ng} \mathrm{FH}$, Tunggal $\mathrm{P}$, Chu WM, et al. Esomeprazole compared with famotidine in the prevention of upper gastrointestinal bleeding in patients with acute coronary syndrome or myocardial infarction. Am J Gastroenterol. 2012;107(3):389-396.

25. Tunggal P, Ng FH, Lam KF, Chan FK, Lau YK. Effect of esomeprazole versus famotidine on platelet inhibition by clopidogrel: A doubleblind, randomized trial. Am Heart J. 2011;162(5):870-874.

26. Hochholzer W, Trenk D, Fromm MF, et al. Impact of cytochrome P450 2C19 loss-of-function polymorphism and of major demographic characteristics on residual platelet function after loading and maintenance treatment with clopidogrel in patients undergoing elective coronary stent placement. J Am Coll Cardiol. 2010;55(22):2427-2434.

27. O'Donoghue ML, Braunwald E, Antman EM, et al. Pharmacodynamic effect and clinical efficacy of clopidogrel and prasugrel with or without a proton-pump inhibitor: An analysis of two randomised trials. Lancet. 2009;374(9694):989-997.

28. Simon T, Steg PG, Gilard M, et al. Clinical events as a function of proton pump inhibitor use, clopidogrel use, and cytochrome P450 2C19 genotype in a large nationwide cohort of acute myocardial infarction: Results from the French Registry of Acute ST-Elevation and Non-ST-Elevation Myocardial Infarction (FAST-MI) registry. Circulation. 2011;123(5):474-482. 